\title{
Resolution in Model-Based Measurement
}

\author{
Adriaan van den Bos, Fellow, IEEE
}

\begin{abstract}
In measurement practice, the concept resolution is usually associated with the ability to distinguish two overlapping components of the same kind in observations. The original concept, Rayleigh's well-known two-point resolution, stems from optics. It is based on the presumed limits of the human visual system to distinguish the images of two closely located point sources in observations of the sum of the images. Modern definitions of resolution, on the other hand, are based on parametric statistical models of the observations. They implicitly assume the use of parameter estimation methods and show that the ultimate limits to resolution are nonsystematic (statistical) and systematic (modeling) errors.
\end{abstract}

Index Terms-Model-based measurement, parameter estimation, resolution.

\section{INTRODUCTION}

$\mathbf{T}$ HE AIM of this paper is to introduce and explain modern model-based definitions of the concept resolution. A further purpose is to review earlier definitions and to discuss their differences with the modern approach.

The earlier approaches are the subject of the Sections II and III of this paper. In Section II, so-called classical definitions of resolution are reviewed. The best known of these is the Rayleigh resolution criterion or limit. It defines the presumed minimum distance at which the overlapping component images of two identical, closely located point sources can still be distinguished by the human visual system. Since this definition supposes this minimum distance proportional to the width of the point images, imaging instruments have better resolving capabilities as their point image, the so-called point spread function, is narrower. Thus, Rayleigh's and related limits to resolution are purely deterministic and instrumental. They are defined by the width of the point-spread function or, equivalently, by its reciprocal, the bandwidth of its Fourier transform called optical system transfer function.

More recent approaches are based on the idea that resolution is limited by noise in the observations. This limitation is incorporated in resolution definitions discussed in Section III, which are based on information theory, decision theory, signal-to-noise ratio (SNR), and asymptotic parameter estimation theory, respectively. Successively, these definitions require an increasing amount of a priori knowledge. In particular, the last definition assumes the parametric model of the point image and the distribution of the observations to be known, and the number of observations to be very large. These conditions are very demanding and seriously limit the applicability.

In Sections IV-X, an attractive modern definition of resolution is explained. It is also based on a parameter estimation ap-

Manuscript received May 29, 2001; revised July 22, 2002.

The author is with the Department of Physics, Delft University of Technology, Delft, The Netherlands (e-mail: a.vandenbos@tnw.tudelft.nl).

Digital Object Identifier 10.1109/TIM.2002.806031

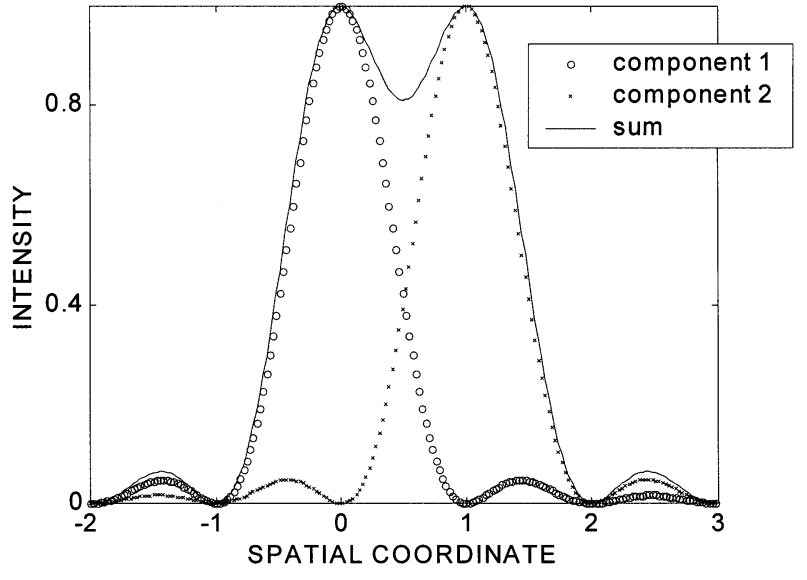

Fig. 1. Rayleigh sinc square model.

proach but does not require asymptoticity. For any estimation method and two-component model, it divides all possible sets of two-component observations into two types: sets from which the components can be resolved or sets from which they cannot. Nonsystematic (statistical) errors and systematic (modeling) errors then determine the probability that a set of observations belongs to the former type, that is, they determine the probability of resolution. The required parameter estimation notions are introduced in Section IV. Sections V and VI are mainly devoted to a numerical example illustrating the actual occurrence of both types of observations. Section VII introduces a singularity theory based criterion specifying to which type a particular set of observations belongs. The numerical computation of the criterion value is the subject of Section VIII. Probability of resolution, discussed in Section IX, is defined using the same criterion. Section X describes generalizations and extensions. Conclusions are drawn in Section XI.

\section{Classical Two-Component Resolution}

Of all resolution criteria, that of Rayleigh is probably oldest and best known [1]. Rayleigh considered two overlapping, incoherent, sinc square images as just resolvable from their sum if the central maximum of the one image coincides with the first zero of the other such as shown in Fig. 1. At the relative minimum in between the peaks, the sum is approximately equal to $81 \%$ of the peak value, and this intensity ratio was thought to be the limit to what the human visual system can perceive. Later, the same ratio was used to define limits to resolvability of images other than sinc square. For all these images, the distance of the images of the peaks corresponding to the $81 \%$ ratio is called the Rayleigh limit. Its main characteristic is that it is smaller as the central peak of the image is narrower.

In optics literature, a number of alternatives to Rayleigh's definition have been proposed. Schuster and Nicholson 
[2, p. 158] require the locations of the peaks to be such that the central peaks do not overlap at all. This implies that for the sinc square image the Schuster Nicholson limit is twice the Rayleigh limit. Sparrow [3] proposes a resolution limit so that both maxima and the relative minimum in between just coincide and a sum image results that has one single peak. This means that the component images are more closely located than at the Rayleigh limit. Simple calculations show that for the sinc square image, the Sparrow limit is 0.83 times the Rayleigh limit.

The three resolution limits described, and similar ones found in the literature, are called classical resolution limits. They have in common that they are in fact measures of the widths of the component images. Since in these classical resolution criteria the component image is the point spread function, resolution is better in the sense of these criteria as this function is narrower. The shape of the point-spread function depends on the shape of the aperture and its spatial transmittance function, the pupil function. Many attempts are described in the literature to make the central peak of the point-spread function narrower by proper choice of the pupil function. Unfortunately, this process, sometimes called apodization [4], always reduces the total amount of light transmitted. Specifically, it reduces the value of the point-spread function at its central maximum and creates side lobes, which make the image more difficult to interpret.

In conclusion, classical resolution limits concern the exact sum of two overlapping known point spread functions. However, if this known two-component model would be fitted with respect to its amplitude and location parameters to the exact observations, a perfect fit and unlimited resolution would result. Therefore, if there are limits to resolution, these must be a consequence of nonsystematic (statistical) and systematic (modeling) errors. The notion that errors and a priori knowledge determine resolution is seen in a number of developments after the classical approach described in the sections to follow.

\section{Statistical Definitions of Resolution}

In the preceding section, it was concluded that errors ought to be included in the quantification of resolution. This section reports a number of attempts in the literature to do so.

The first approach to be discussed is the use of the information theoretical concept channel capacity, which is the number of degrees of freedom a system can transmit. Cox and Sheppard [5] derived a general expression for the channel capacity of an imaging system as a function of spatial bandwidth and SNR. If the channel capacity of an imaging system is assumed invariant, this expression shows that increase of spatial bandwidth, that is, resolution can only be achieved at the expense of the SNR, that is, precision of the result. This result in the spatial domain is comparable to the classical frequency-domain result in spectral analysis where the product of the variance and the resolution of a spectral estimate is invariant for a particular set of observations [6].

A related straightforward approach is to directly associate resolution with the SNR of the image observations. The literature concerned is vast. A typical example of this approach is
[7] where the SNR in the frequency domain is used. If the bandwidth of the imaged object is assumed to be smaller than that of the noise, the frequency is determined where the smallest SNR considered acceptable is attained. The reciprocal of this frequency is used as the resolution in the spatial domain. Other SNR-based methods employ a so-called resolution scale. This is a constant of the order of the Rayleigh limit. The precision of location is then equal to the ratio of the resolution scale to the SNR. Unfortunately, tools like the resolution scale are merely rules of thumb neither intended nor suitable to provide insight in what the fundamental limits to resolution are.

Various authors report application of decision theory to resolution of overlapping component functions in observations. An example is [8]. Resolution is then seen as correctly deciding that there are two components present in noisy two-component observations instead of only one. The probability of a correct decision is proposed as a measure of resolution. The decision theoretical approach is operational in the sense that it can be applied to actual two-component observations. However, it requires a correct component model and correct statistical properties of the observations. This may make application problematic. On the other hand, the decision theoretical approach is highly illustrative of a number of aspects of resolution. First, it illustrates the dependence of resolution on the statistical properties of the observations since the probability of a wrong decision will increase as the observations are noisier. Second, in this approach, it is assumed that noisy two-component observations may be divided in one-component-like or two-component-like observations. The usefulness of this idea will be demonstrated in Sections V-X.

As a final example of statistical definitions of resolution found in the literature, asymptotic parameter estimation methods are briefly mentioned. Examples are [9] and [10]. These methods express resolution in terms of the statistical precision with which the locations and the amplitudes of the components can be estimated. For the computation of the precision, the standard deviation is used that is attained asymptotically by maximum likelihood estimators. This implies that the probability density function of the observations must be known and the number of observations must be very large. In addition, these computations require the component function to be exactly known. These conditions seriously limit the applicability. The importance of this approach is that it relates resolution to parametric models and parameter estimation. These subjects will briefly be reviewed in the next section, and the resolution of parameter estimation methods without the restrictive conditions of the asymptotic methods will be the subject of the Sections $\mathrm{V}-\mathrm{X}$.

\section{ElEMENTS OF PARAMETER ESTIMATION}

The Rayleigh two-component model has three parameters only: both locations of the components and the common amplitude. Therefore, these are the only quantities to be estimated to fully characterize the two-component observations. Generally, observations made under the same conditions differ from experiment to experiment as a result of nonsystematic errors. The statement that observations are "described by the Rayleigh 
model" means that a properly parameterized Rayleigh model exactly describes the expectations of the observations [11]. Replacing the expectations present in the joint probability density function of the observations by the model defines the dependence of the probability density function on the parameters. If, next, the available observations are substituted for the corresponding independent variables of the probability density function, the resulting expression as a function of the parameters is called the likelihood function of the parameters. It is a measure of the probability of observations like the ones substituted given the parameters. In what follows, the logarithm of the likelihood function, the log-likelihood function, will be denoted by $q(t)$ where $t$ is the vector of parameters.

The maximum likelihood estimates of the parameters are defined as the parameter values maximizing the likelihood function [12]-[14]. The likelihood function for independent and identically normally distributed errors is equivalent to the ordinary least squares criterion. In practice, this criterion is also used if the observations are distributed otherwise. To include this, and other practical cases, in what follows, the assumption will be made that the parameters of the two-component model are estimated by maximizing a likelihood function that is not necessarily the likelihood function corresponding to the probability density of the observations. Also, the parametric model used may be different from the expectations of the observations. This modeling error is mentioned since as will be seen in what follows, this may influence the resolving of the components from the observations.

\section{IRRESOLVABLE COMPONENT LOCATION PARAMETERS}

The purpose of the numerical example presented in this section is to show that resolution is dependent on the particular realization of the observations.

Example 1: Suppose that in an experiment the expectations of the observations are a two-component model described by

$$
f_{n}(\theta)=\alpha\left[h_{n}\left(\beta_{1}\right)+h_{n}\left(\beta_{2}\right)\right]
$$

where the vector of parameters $\theta$ is defined as $\left(\alpha \beta_{1} \beta_{2}\right)^{T}$

$$
h_{n}\left(\beta_{k}\right)=\exp \left[-\frac{1}{2}\left(x_{n}-\beta_{k}\right)^{2}\right]
$$

is the Gaussian component function with $\beta_{k}$ as location parameter, and $x_{n}$ is the $n$th measurement point. Specifically, suppose that $x_{n}=-2.525+0.5(n-1), n=1, \ldots, 11$ and $\theta=\left(\begin{array}{lll}\alpha & \beta_{1} & \beta_{2}\end{array}\right)^{T}=\left(\begin{array}{lll}5000 & 0 & 0.15\end{array}\right)^{T}$. This choice of the $x_{n}$ avoids undesirable symmetries in the location of the measurement points. Fig. 2 shows the function and the locations of the measurement points. Furthermore, suppose that the observations are independent and Poisson distributed and that the corresponding maximum likelihood estimator is used for the estimation of $\theta$. Then, it is not difficult to show [15] that the maximum likelihood estimator for $\alpha$ may be expressed in closed form in those for $\beta_{1}$ and $\beta_{2}$. If this expression is subsequently substituted back into the likelihood function, a function of $b_{1}$ and $b_{2}$, respectively, corresponding to the parameters $\beta_{1}$ and $\beta_{2}$, is obtained. The contours of this function are shown in Figs. 3 and 4 for two different realizations of the observations. In both figures, the horizontal and vertical coordi-

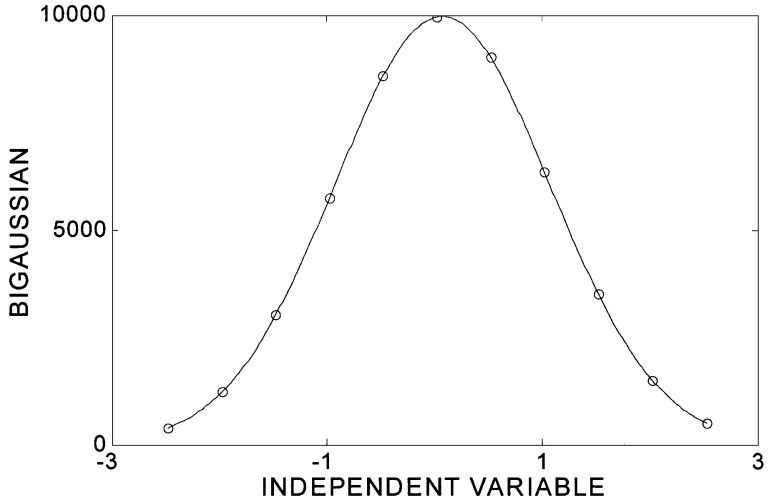

Fig. 2. Measurement points on Bigaussian function of Example 1.

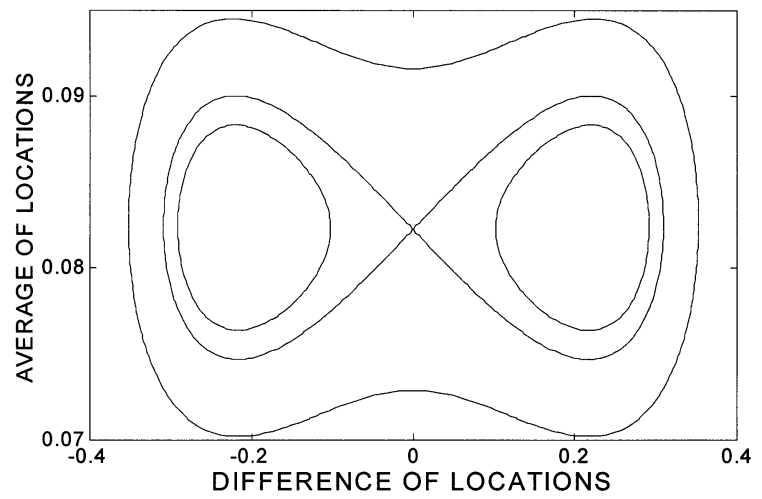

Fig. 3. Two maxima, one saddle point structure of the likelihood function of Example 1.

nates are $b_{1}-b_{2}$ and $\left(b_{1}+b_{2}\right) / 2$, respectively. These coordinates are, therefore, the difference and the average of the locations of the components of the two-component model, respectively. Both Figs. 3 and 4 are symmetric in the $\left(b_{1}+b_{2}\right) / 2$-axis since $b_{1}$ and $b_{2}$ are interchangeable. However, the structure, defined as the pattern of stationary points, is essentially different. The likelihood function of Fig. 3 has a maximum on either side of the $\left(b_{1}+b_{2}\right) / 2$-axis and has a saddle point on this axis. The coordinates of the left-hand maximum correspond to about $\left(b_{1}, b_{2}\right)=(-0.027,0.191)$ which are estimates of the true values $\left(\beta_{1}, \beta_{2}\right)=(0,0.15)$. The saddle point on the $\left(b_{1}+b_{2}\right) / 2$-axis corresponds to $\left(b_{1}, b_{2}\right)=(0.0822,0.0822)$. Since these values for $b_{1}$ and $b_{2}$ are exactly equal, the components coincide and the corresponding model is a one-component model of the same parametric family. More specifically, it is not difficult to show that this is the one-component model best fitting to the observations [15]. Fig. 4 differs from Fig. 3 in two respects. First, the maxima on either side of the $\left(b_{1}+b_{2}\right) / 2$-axis have disappeared. Second, the stationary point on this axis has become a maximum instead of a saddle-point and, therefore, represents the maximum likelihood solution. The conclusion is that this maximum likelihood solution is a one-component solution instead of a two-component solution. Irrespective of its nature, the stationary point on the $\left(b_{1}+b_{2}\right) / 2$-axis will from now on be called the one-component stationary point.

A conclusion from Example 1 is that two-component observations generated under the same conditions may be one-component-like or two-component-like. It is clear that, if the obser- 


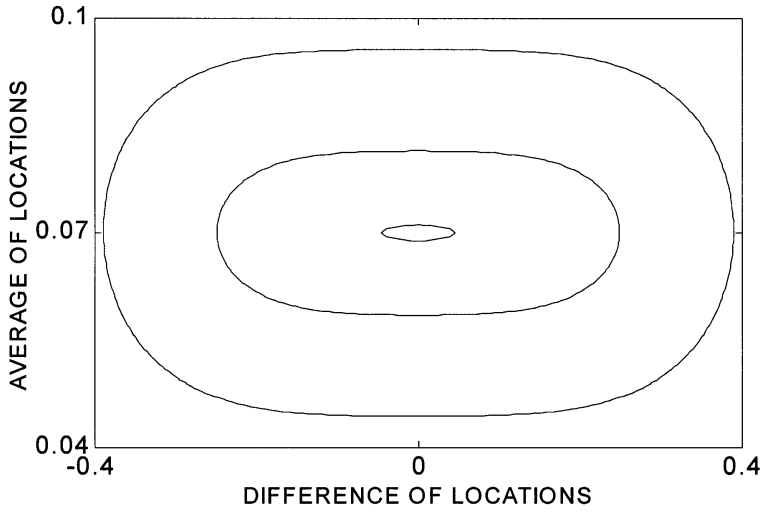

Fig. 4. One maximum structure of the likelihood function of Example 1.

vations are one-component-like, the distinct parameters $\beta_{1}$ and $\beta_{2}$ are no longer resolved from the observations since the solutions coincide. The question now arises what distinguishes both types of observations? This question will be addressed in the next section.

\section{RESOlution AS A PROPERTY OF OBSERVATIONS}

Example 1 showed that the irresolvability of component location parameters or, equivalently, the components, is caused by structural change. Structural change is a subject of singularity theory which, combined with its applications, is also called catastrophe theory [16], [17]. To illustrate a number of relevant concepts of those theories, first a numerical example is presented. In this example, the concept (Euclidean) space of the observations is used. This is the Euclidean space with the observations as coordinates. Its dimension is the number of observations.

Example 2: Suppose that a convex combination is made of the two sets of observations of Example 1. This means that the corresponding observations in both sets are added with weights $1-\lambda$ and $\lambda$ with $0 \leq \lambda \leq 1$ to form a new set of "observations." Then, if $\lambda=0$, the set of observations thus formed corresponds to Fig. 3, while the one for $\lambda=1$ corresponds to Fig. 4. Each of both sets is a point in the space of observations, and the straight line connecting them represents all convex combinations. Then, if $\lambda$ is increased from zero on, eventually on this line there is a point where the solutions for the locations just coincide. This is illustrated in Fig. 5 showing the solutions for the locations as a function of $\lambda$. The figure also shows that once the solutions coincide, they continue to do so for increasing $\lambda$.

In singularity theory, the set of all points in the space of the observations where the solutions for the locations just coincide and the structure changes is called a bifurcation set. It will be shown that the bifurcation set concerned is a hypersurface. This is a subspace of codimension 1 on the space of observations. Subspaces of codimension 1 have the property that they are described by a single equation and divide the space in which they are located into two distinct parts. Examples are a point on a line, or a line in the plane. In the resolution problems considered, the bifurcation set divides the space of possible observations into two parts. The observations in the one part correspond to distinct values for the locations of the components, that is, they

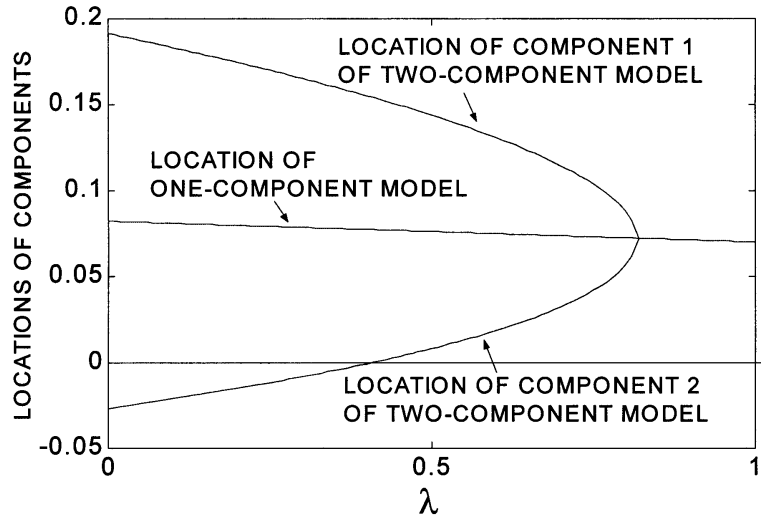

Fig. 5. Solutions for the locations in Example 2.
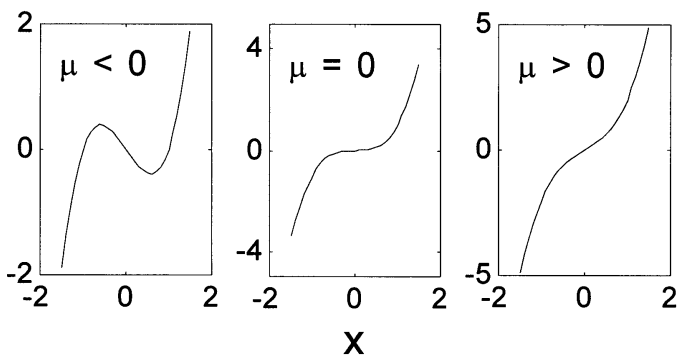

Fig. 6. Structures of the function in Example 3 for different values of the parameter $\mu$.

are two-component-like. The observations in the complementary part of the space of observations correspond to coinciding solutions for the locations, that is, they are one-component-like. Therefore, the bifurcation set defines from which sets of observations the components can be resolved and from which they cannot. It is, therefore, important to be able to compute the bifurcation set or to determine in a simple way on which side of the bifurcation set, a set of observations is located. How this can be done is the subject of the subsequent sections.

\section{Classifying OBSERVATIONS}

To find the bifurcation set, the following result from singularity theory is used: structural change of a function only occurs if one or more stationary points of the function become degenerate. If one or more eigenvalues of the Hessian matrix evaluated at a stationary point are equal to zero ("vanish"), the stationary point is called degenerate. In what follows, it will be assumed that only one of the eigenvalues may vanish since this covers the applications in this study. To illustrate degeneracy of this kind, first an example is presented.

Example 3: In Fig. 6, the function $\mu x+(1 / 3) x^{3}$ is shown for $\mu=-1,0$, and 1 , respectively. For $\mu=-1$, and for all other negative $\mu$, the function has one relative maximum and one relative minimum. These stationary points merge to form one degenerate stationary point at $x=0$ if $\mu$ vanishes. Subsequently, for $\mu$ positive, this stationary point disappears. In this simple case, the bifurcation set consists of $\mu=0$ since this is the set of all parameter values for which a degenerate stationary point occurs. 
Different from the cubic polynomial in one variable in this example, the log-likelihood functions studied are functions of the three variables $a, b_{1}, b_{2}$ or, equivalently, $a,\left(b_{1}+b_{2}\right) / 2$ and $b_{1}-b_{2}$. A further difference is that the log-likelihood functions are not polynomials. However, in bifurcation theory it is shown that for the analysis of structural change of a function it is sufficient to study a Taylor polynomial in the coordinates in which the structural change takes place. In the problem at hand this is only the coordinate $b_{1}-b_{2}$. This is demonstrated in Figs. 3 and 4 where in the $\left(b_{1}+b_{2}\right) / 2$ coordinate the function remains maximum but in the $b_{1}-b_{2}$ coordinate changes from a two maxima, one minimum structure into a single maximum. Singularity theory also prescribes the required degree of this Taylor polynomial.

In [15], the Taylor polynomial in $b_{1}-b_{2}$ and around the one-component stationary point is derived for log-likelihood functions of the parameters of $t=\left(\begin{array}{lll}a & b_{1} & b_{2}\end{array}\right)^{T}$ of the two-component model $f_{n}(t)$. The result is described by

$$
\delta_{2} \Delta^{2}+\delta_{4} \Delta^{4}
$$

with $\Delta=b_{1}-b_{2}$. This polynomial has two equivalent maxima and a minimum, at the origin, in between if $\delta_{2}>0$. It has a single maximum at the origin if $\delta_{2} \leq 0$. This maximum is degenerate if $\delta_{2}=0$. Therefore, the structures occurring are those depicted in Figs. 3 and 4, respectively. Specifically, the single maximum occurring if $\delta_{2} \leq 0$ is located at the origin, that is, at $\Delta=b_{1}-b_{2}=0$. Then the solutions for both locations coincide and the components are not resolved. If $\delta_{2}>0$, the likelihood function has a saddle point at the origin and two equivalent maxima for $\Delta=b_{1}-b_{2} \neq 0$. Then the solutions for the locations of the components are different and the components are resolved. The conclusion is that the sign of $\delta_{2}$ is the resolution criterion.

\section{NumericAl COMPUTATION OF THE CRITERION VALUE}

In [15], it is shown that the sign of $\delta_{2}$ is the sign of the quantity

$$
D=\sum_{n} \frac{\partial q}{\partial f_{n}} h_{n}^{(2)}(\hat{b})
$$

where $f_{n}=f_{n}(t), q=q(t)$, and $h_{n}^{(2)}(\hat{b})$ is the second-order derivative of $h_{n}(b)$ with respect to $b$. The expression (4) is evaluated at the one-component stationary point $\hat{t}=(\hat{a} \hat{b} \hat{b})^{T}$. Notice that this criterion is applicable to any log-likelihood function and component function.

The procedure for the computation of the criterion (4) is as follows. First, the maximum likelihood solutions $\hat{a}$ and $\hat{b}$ for the parameters $a$ and $b$ of the one-component model $2 a h_{n}(b)$ are estimated from the available observations. The estimates are substituted in (4). The sign of the result shows if distinct estimates for $\beta_{1}$ and $\beta_{2}$ are obtained or not. Thus, for a general class of log-likelihood functions and component functions, the quantity $D$ is an unambiguous criterion for resolvability.

In [15], it is also shown that the three equations

$$
\sum_{n} \frac{\partial q}{\partial f_{n}} h_{n}^{(\ell)}(\hat{b})=0 \quad \text { for } \ell=0,1,2
$$

define the bifurcation set. They are obtained by equating both the gradient of the log-likelihood function with respect to $a$ and $b$ and the quantity $D$ defined by (4) to zero. Thus it is certain that $\hat{t}=(\hat{a} \hat{b} \hat{b})^{T}$ is the one-component stationary point and that this point is singular. Notice that (5) represents three equations in the $N$ observations and the parameters $\hat{a}$ and $\hat{b}$. Hence, after hypothetical elimination of both parameters, a single equation in the $N$ observations results. Therefore, the bifurcation set has codimension 1 and divides the space of observations into two regions corresponding to one-component-like and two-component-like observations, respectively.

\section{Probability of Resolution}

If the observations are statistical, the sign of the criterion $D$ becomes a stochastic variable. Then, the probability that $D$ is positive is the probability of resolution. In the space of the observations, the points representing the sets of observations are distributed about the point representing the expectations of the observations. The probability of resolution is the probability that a point representing a set of observations is on the side of the bifurcation set corresponding to distinct component locations. The probability of resolution is, therefore, determined by the location of the point representing the expectations relative to the bifurcation set and by the distribution of the observations about this point. Equations (4) and (5) show that the bifurcation set or, equivalently, the resolution criterion depends on the chosen likelihood function, on the chosen component function, and on the number of measurement points and their locations. The chosen component function may be wrong which means that it is different from the function describing the components of the expectations of the observations. Then it is even possible that the point representing the expectations is on the side of the bifurcation corresponding to nonresolution This illustrates the influence of modeling errors, that is, systematic errors on resolution and on the probability of resolution. For illustrative examples of the influence of systematic and nonsystematic errors on resolution, see [15].

Equation (5) shows that the bifurcation set is specific for the chosen component function. This function may describe instrumental response, such as the point spread function or the impulse response. Then the bifurcation set is characteristic of the kind of instrument used. Since the distribution of the observations and the bifurcation set combined determine the probability of resolution, the instrument influences this probability. However, as the systematic and nonsystematic errors are smaller, the probability of resolution increases, no matter the nature of the component function, that is, the characteristics of the instrument.

\section{Generalizations And EXTENSIONS}

The resolution definition using the criterion $D$ defined by (4) has been generalized in a number of respects. First, it may be shown to apply if the amplitudes of the components are different. Also, a parametric background function may be added to the two-component model, for example, a trend, an offset, 
or a day-and-night cycle with one or more unknown parameters. Furthermore, the resolution criterion may, with slight modifications, also be used for component functions that are functions of two or more variables. Examples are two-dimensional components in optics and electron optics, such as Airy patterns. Moreover, in optical applications, it may be used in the presence of partial coherence of the components. Finally, the analysis presented is not limited to two-peak models but also includes models like two sinusoids with closely located frequencies or biexponentials with little differing decays. For a survey of these generalizations and extensions, see [15].

\section{CONCLUSION}

The first part of this paper is a sketch of the development of the concept of resolution. Classical definitions like Rayleigh's are fully based on properties of the imaging instrument and are deterministic. Later definitions, inspired by information theory, decision theory, or asymptotic parameter estimation theory, depend on the statistical properties of the observations. Finally, a relatively recent alternative definition is presented also inspired by parameter estimation but avoiding unrealistic assumptions, in particular asymptoticity.

The second part of the paper introduces this alternative definition and outlines its theoretical basis. Its main characteristic is that it is much more general than the existing definitions since it applies to any number of observations, any likelihood function, and component function used. Moreover, the errors may be statistical fluctuations of the observations or systematic, that is, modeling errors.

\section{REFERENCES}

[1] J. W. Strutt and L. Rayleigh, Scientific Papers by John William Strutt, Baron Rayleigh. Cambridge, U.K.: Cambridge Univ. Press, 1902, vol. 3 (1887-1892), ch. Wave Theory of Light, pp. 47-189.

[2] A. Schuster and J. W. Nicholson, An Introduction to the Theory of Optics. London, U.K.: Arnold, 1924.

[3] C. M. Sparrow, "On spectroscopic resolving power," Astrophys. J., vol. 44, pp. 76-86, 1916.

[4] P. Jacquinot and B. Roizen-Dossier, "Apodization," in Progress in Optics, E. Wolf, Ed. Amsterdam, The Netherlands: North-Holland, 1964, vol. 3.
[5] I. J. Cox and C. J. R. Sheppard, "Information capacity and resolution in an optical system," J. Opt. Soc. Amer. A, vol. 3, pp. 1152-1158, 1986.

[6] R. B. Blackman and J. W. Tukey, The Measurement of Power Spectra from the Point of View of Communications Engineering. New York: Dover, 1958.

[7] P. S. Idell and A. Webster, "Resolution limits for coherent optical imaging: Signal-to-noise analysis in the spatial-frequency domain," $J$. Opt. Soc. Amer. A, vol. 9, pp. 43-56, 1992.

[8] J. L. Harris, "Resolving power and decision theory," J. Opt. Soc. Amer, vol. 54, pp. 606-611, 1964.

[9] T. Orhaug, "On the resolution of imaging systems," Optica Acta, vol. 16, pp. 75-84, 1969.

[10] E. Bettens, D. van Dyck, A. J. den Dekker, J. Sijbers, and A. van den Bos, "Model-based two-object resolution from observations having counting statistics," Ultramicroscopy, vol. 77, pp. 37-48, 1999.

[11] A. van den Bos, "Measurement errors," in Wiley Encyclopedia of Electrical and Electronics Engineering, J. G. Webster, Ed. New York: Wiley, 1999, vol. 12, pp. 448-459.

[12] A. Stuart and J. K. Ord, Kendall's Advanced Theory of Statistics, 6th ed. London, U.K.: Arnold, 1994, vol. 1, Distribution theory.

[13] A. Stuart, J. K. Ord, and S. Arnold, Kendall's Advanced Theory of Statistics, 6th ed. London, U.K.: Arnold, 1999, vol. 2A, Classical inference and the linear model.

[14] A. van den Bos, "Parameter estimation," in Handbook of Measurement Science, P. H. Sydenham, Ed. Chicester, U.K.: Wiley, 1982, vol. 1, ch. 8, pp. 331-377.

[15] A. van den Bos and A. J. den Dekker, "Resolution reconsidered-Conventional approaches and an alternative," in Advances in Imaging and Electron Physics, P. W. Hawkes, Ed. San Diego, CA: Academic, 2001, vol. 117 , pp. 241-360.

[16] T. Poston and I. N. Stewart, Catastrophe Theory and Its Applications. London, U.K.: Pitman, 1978.

[17] P. T. Saunders, An Introduction to Catastrophe Theory. Cambridge, U.K.: Cambridge Univ. Press, 1980

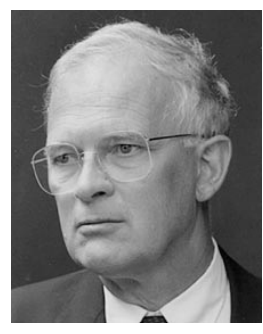

Adriaan van den Bos (SM'94-F'00) received the M.S. degree in applied physics and the D.Tech.Sc. degree from the Delft University of Technology, Delft, The Netherlands, in 1962 and 1974, respectively.

From 1962 to 1964, he was with the Institute of Perception of the Royal Defence Research Council, Soesterberg, The Netherlands, working on instrumentation problems. In 1964, he joined the Department of Applied Physics, Delft University of Technology, where he is currently Full Professor. His main research interest is in applying parameter estimation to measurement problems in various fields of physics. 